International Journal of Difference Equations (IJDE).

ISSN 0973-6069, Volume 16, Number 1, (2021). 81-93

(C) Research India Publications

https://dx.doi.org/10.37622/IJDE/16.1.2021.81-93

\title{
Subclasses of Univalent Functions Involving Modified Sigmoid Function
}

\author{
${ }^{1}$ Olubunmi A. Fadipe-Joseph, ${ }^{2,3}$ M. O. Oluwayemi, ${ }^{1}$ E. O. Titiloye \\ ${ }^{1}$ Department of Mathematics, University of Ilorin, P.M.B. 1515, Ilorin, Nigeria. \\ ${ }^{2}$ Landmark University SDG 4 (Quality Education Research Group), Omu-Aran, Nigeria. \\ ${ }^{3}$ Department of Mathematics, Landmark University, P.M.B. 1001, Omu-Aran, Nigeria.
}

\begin{abstract}
The authors obtained some geometric results on certain new classes of analytic functions involving sigmoid function defined by Fadipe-Joseph et. al. 2016 as $T_{\gamma}(\lambda, \beta, \alpha, \mu)$. Extreme point property, radius of starlikeness and convexity, convolution property and Fekete-Szego inequality for the class were proved.
\end{abstract}

Keywords: Sălăgean operator, modified sigmoid function, radius of starlikeness and convexity, convolution property and Fekete-Szego inequality.

AMS Mathematics Subject Classification (2010): 30C45, 30C50.

\section{INTRODUCTION}

The study of subclasses of analytic and univalent functions in geometric theory and applications is a link between geometry and analysis with wide range of interest among function theorists in recent time. Subclasses of analytic and univalent functions can be as many as researchers who are of interest in the area (see [1], [2], [3] and [4]). In this paper however, using Sălăgean differential operator involving modified sigmoid function $\gamma(s)$, we investigate some geometric properties of the class $T_{\gamma}(\lambda, \beta, \alpha, \mu)$ introduced in [1].

Let $T_{\gamma}$ denote the class of functions of the form

$$
f_{\gamma}(z)=z-\sum_{k=2}^{\infty} \gamma(s) a_{k} z^{k} ; \quad a_{k} \geq 0, \quad \gamma(s)=\frac{2}{1+e^{-s}}
$$


and

$$
g_{\gamma}(z)=z-\sum_{k=2}^{\infty} \gamma(s) b_{k} z^{k} ; \quad b_{k} \geq 0
$$

which are analytic and univalent in the unit disk $U=\{|z|: z \leq 1\}$.

Then $f_{\gamma}(z)$ and $g_{\gamma}(z)$ belong to class $T_{\gamma}$.

If for convenience, we set $T_{\gamma}=T_{1}$ we see that $T_{1}=T$, is the usual class of the form $f(z)=z-\sum_{k=2}^{\infty} a_{k} z^{k} ; \quad a_{k} \geq 0$ which is analytic in the open unit disk $U$.

We define an identity function as

$$
e_{\gamma}(z)=z ; a_{k}=0 \text { for all } k \geq 2 \text { but } \gamma \neq 0
$$

\subsection{Convolution or Hardamard Product:}

Given two analytic function $f_{\gamma}(z)$ and $g_{\gamma}(z)$ in $T_{\gamma}$ where $f_{\gamma}(z)$ and $g_{\gamma}(z)$ are given by (1.1) and (1.2) respectively.

Convolution of $f_{\gamma}(z)$ and $g_{\gamma}(z)$ are defined as

$$
\left(f_{\gamma} * g_{\gamma}\right)=z-\sum_{k=2}^{\infty} \gamma(s) a_{k} b_{k} z^{k}=\left(g_{\gamma} * f_{\gamma}\right) ; \quad a_{k} b_{k} \geq 0
$$

\subsection{Starlikeness, Convexity and Close-to-convexity:}

A function $f(z)$ defined by (1.1) is said to be starlike of order $\delta$ if

$$
\operatorname{Re}\left\{\frac{z f^{\prime}(z)}{f(z)}\right\}>\delta ; \quad z \in U
$$

for some $\delta(0 \leq \delta \leq 1)$.

In the same way, a function $f(z)$ defined by (1.1) is said to be convex of order $\delta$ if and only if $z f^{\prime}(z)$ is starlike of order $\delta$. In other words, if

$$
\operatorname{Re}\left\{1+\frac{z f^{\prime \prime}(z)}{f^{\prime}(z)}\right\}>\delta ; \quad z \in U
$$

for some $\delta(0 \leq \delta \leq 1)$.

Furthermore, a function $f(z)$ defined by (1.1) is said to be close-to-convex of order $\delta$ if

$$
\operatorname{Re}\left\{z f^{\prime}(z)\right\}>\delta ; \quad z \in U
$$

for some $\delta(0 \leq \delta \leq 1)$. 


\section{PRELIMINARY RESULTS}

\subsection{Sălăgean Differential Operator Involving Modified Sigmoid Function}

Definition 2.1 Sălăgean Differential Operator Involving Modified Sigmoid Function

$$
D^{n} f_{\gamma}(z)=\gamma^{n}(s) z+\sum_{k=2}^{\infty} \gamma^{m}(s) k^{n} a_{k} z^{k} ; m=n+1
$$

for details see [1].

Definition 2.2 A function $f_{\gamma} \in T_{\gamma}$ defined by (1.1) belongs to the class $T_{\gamma}(\lambda, \beta, \alpha, \mu)$ if

$$
\left|\frac{\frac{D^{n+1} f_{\gamma}(z)}{D^{n} f_{\gamma}(z)}-\mu}{\left(\frac{D^{n+1} f_{\gamma}(z)}{D^{n} f_{\gamma}(z)}+\lambda\right)-2 \alpha\left(\frac{D^{n+1} f_{\gamma}(z)}{D^{n} f_{\gamma}(z)}-\mu\right)}\right|>\beta \quad(z \in U)
$$

for $|z|<1,0<\lambda \leq 1, \gamma(s)=\frac{2}{1+e^{-s}}$ (i.e. $\left.\gamma \neq 0\right), 0<\beta \leq 1, \frac{1}{2} \leq \alpha \leq 1, \mu \geq 1$, $n \in N_{0}=N \cup\{0\} \quad$ See $[1]$.

\section{Lemma 2.1 [1]}

Let $f_{\gamma}(z) \in T_{\gamma}$ defined as $f_{\gamma}(z)=z-\sum_{k=2}^{\infty} \gamma(s) a_{k} z^{k}\left(a_{k} \geq 0\right.$ and $\left.\gamma \neq 0\right)$. If $f_{\gamma} \in T_{\gamma}(\lambda, \beta, \alpha, \mu)$, then

$$
\begin{gathered}
\sum_{k=2}^{\infty} \gamma(s) k^{n}\left[\gamma(s) k[1-\beta(1-2 \alpha)]-\beta\left(\lambda+2 \alpha \mu+\frac{1}{\beta}\right)\right]\left|a_{k}\right| \\
<\gamma(s)(1-\beta)+\beta[2 \alpha(\gamma(s)-\mu)-\lambda]-\mu .
\end{gathered}
$$

\section{MAIN RESULTS}

Theorem 3.1 Extreme points for class $T_{\gamma}(\lambda, \beta, \alpha, \mu)$.

If a function $f_{\gamma}(z)$ defined by (1.1) belongs to the class $T_{\gamma}(\lambda, \beta, \alpha, \mu)$.

Let $f_{1}(z)=z$ and

$$
f_{\gamma}(z)=\frac{\gamma(s)(1-\beta)+\beta[2 \alpha(\gamma(s)-\mu)-\lambda]-\mu}{\gamma(s) k^{n}\left[\gamma(s) k[1-\beta(1-2 \alpha)]-\beta\left(\lambda+2 \alpha \mu+\frac{1}{\beta}\right)\right]} z^{k}, \quad k \geq 2 .
$$

The function $f_{\gamma} \in T_{\gamma}(\lambda, \beta, \alpha, \mu)$ if and only if it can be expressed in the form

$$
f_{\gamma}(z)=\sum_{k=1}^{\infty} \Psi_{k} f_{k}(z)
$$

where $\Psi_{k} \geq 0$ and $\sum_{k=1}^{\infty} \Psi_{k}=1$. 
Proof. Let $f_{\gamma}(z)=\sum_{k=1}^{\infty} \Psi_{k} f_{k}(z), \Psi_{k} \geq 0, k=1,2, \ldots$ with

$$
\begin{gathered}
\sum_{k=1}^{\infty} \Psi_{k}=1 \\
f_{\gamma}(z)=\sum_{k=1}^{\infty} \Psi_{k} f_{k}(z) \\
=\Psi_{1} f_{1}(z)+\sum_{k=2}^{\infty} \Psi_{k} f_{k}(z) \\
=\Psi_{1}(z)+\sum_{k=2}^{\infty} \Psi_{k}\left\{z-\frac{\gamma(s)(1-\beta)+\beta[2 \alpha(\gamma(s)-\mu)-\lambda]-\mu}{\gamma(s) k^{n}\left[\gamma(s) k[1-\beta(1-2 \alpha)]-\beta\left(\lambda+2 \alpha \mu+\frac{1}{\beta}\right)\right]} z^{k}\right\}
\end{gathered}
$$

that is,

$$
f_{\gamma}(z)=z-\sum_{k=2}^{\infty} \Psi_{k} \frac{\gamma(s)(1-\beta)+\beta[2 \alpha(\gamma(s)-\mu)-\lambda]-\mu}{\gamma(s) k^{n}\left[\gamma(s) k[1-\beta(1-2 \alpha)]-\beta\left(\lambda+2 \alpha \mu+\frac{1}{\beta}\right)\right]} z^{k} .
$$

Then,

$$
\begin{aligned}
f_{\gamma}(z)= & \sum_{k=2}^{\infty} \Psi_{k} \frac{\gamma(s)(1-\beta)+\beta[2 \alpha(\gamma(s)-\mu)-\lambda]-\mu}{\gamma(s) k^{n}\left[\gamma(s) k[1-\beta(1-2 \alpha)]-\beta\left(\lambda+2 \alpha \mu+\frac{1}{\beta}\right)\right]} \\
& \times \frac{\gamma(s) k^{n}\left[\gamma(s) k[1-\beta(1-2 \alpha)]-\beta\left(\lambda+2 \alpha \mu+\frac{1}{\beta}\right)\right]}{\gamma(s)(1-\beta)+\beta[2 \alpha(\gamma(s)-\mu)-\lambda]-\mu}
\end{aligned}
$$

So that

$$
\sum_{k=2}^{\infty} \Psi_{k}=1-\Psi_{1} \leq 1
$$

In other words,

$$
f_{\gamma}(z)=\Psi_{1}+\sum_{k=2}^{\infty} \Psi_{k}=1 \Rightarrow 1-\Psi_{1} \leq 1
$$

So by Lemma 2.1, $f_{\gamma}(z) \in T_{\gamma}(\lambda, \beta, \alpha, \mu)$.

Conversely, assume that the function $f_{\gamma}(z)$ defined by (1.1) belongs to the class $T_{\gamma}(\lambda, \beta, \alpha, \mu)$, then by Lemma 2.1,

$$
a_{k} \leq \frac{\gamma(s)(1-\beta)+\beta[2 \alpha(\gamma(s)-\mu)-\lambda]-\mu}{\gamma(s) k^{n}\left[\gamma(s) k[1-\beta(1-2 \alpha)]-\beta\left(\lambda+2 \alpha \mu+\frac{1}{\beta}\right)\right]} \quad(k \geq 2) .
$$


Setting

$$
\Psi_{k} \leq \frac{\gamma(s) k^{n}\left[\gamma(s) k[1-\beta(1-2 \alpha)]-\beta\left(\lambda+2 \alpha \mu+\frac{1}{\beta}\right)\right] a_{k}}{\gamma(s)(1-\beta)+\beta[2 \alpha(\gamma(s)-\mu)-\lambda]-\mu}
$$

and

$$
\Psi_{1}=1-\sum_{k=2}^{\infty} \Psi_{k}
$$

So that

$\sum_{k=1}^{\infty} \Psi_{k} f_{k}=\frac{\{\gamma(s)(1-\beta)+\beta[2 \alpha(\gamma(s)-\mu)-\lambda]-\mu\}\left\{\gamma(s) k^{n}\left[\gamma(s) k[1-\beta(1-2 \alpha)]-\beta\left(\lambda+2 \alpha \mu+\frac{1}{\beta}\right)\right]\right\}}{\left\{\gamma(s) k^{n}\left[\gamma(s) k[1-\beta(1-2 \alpha)]-\beta\left(\lambda+2 \alpha \mu+\frac{1}{\beta}\right)\right]\right\}\{\gamma(s)(1-\beta)+\beta[2 \alpha(\gamma(s)-\mu)-\lambda]-\mu\}}$.

We thus notice that we can express $f_{k}(z)$ in the form (3.1).

Therefore, $f_{\gamma}(z)=\sum_{k=1}^{\infty} \Psi_{k} f_{k}$ which completes the proof.

Fekete-Szegǒ inequality for the class $T_{\gamma}(\lambda, \beta, \alpha, \mu)$

In this section, we use the values of $a_{2}$ and $a_{3}$ given by Lemma 2.1 to establish the Fekete-Szegǒ inequality for functions $f_{\gamma}(z)$ belonging to the class $T_{\gamma}(\lambda, \beta, \alpha, \mu)$.

Theorem 3.2 Fekete-Szegǒ inequality for class $T_{\gamma}(\lambda, \beta, \alpha, \mu)$.

If a function $f(z) \in T_{\gamma}$ defined by (1.1) belongs to the class $T_{\gamma}(\lambda, \beta, \alpha, \mu)$ and $\mu \in R$. Then,

$$
\left|a_{3}-\sigma a_{2}^{2}\right| \leq \frac{B_{3}\left[B_{2}^{2}-\sigma B_{3} B_{1}\right]}{B_{1} B_{2}^{2}} .
$$

Proof: From Lemma 2.1,

$$
\begin{gathered}
a_{2} \leq \frac{\gamma(s)(1-\beta)+\beta[2 \alpha(\gamma(s)-\mu)-\lambda]-\mu}{\gamma(s) 2^{n}\left[2 \gamma(s)[1-\beta(1-2 \alpha)]-\beta\left(\lambda+2 \alpha \mu+\frac{1}{\beta}\right)\right]} \quad \text { and } \\
a_{3} \leq \frac{\gamma(s)(1-\beta)+\beta[2 \alpha(\gamma(s)-\mu)-\lambda]-\mu}{\gamma(s) 3^{n}\left[3 \gamma(s)[1-\beta(1-2 \alpha)]-\beta\left(\lambda+2 \alpha \mu+\frac{1}{\beta}\right)\right]}
\end{gathered}
$$

So that

$$
\begin{gathered}
a_{3}-\sigma a_{2}^{2}=\frac{\gamma(s)(1-\beta)+\beta[2 \alpha(\gamma(s)-\mu)-\lambda]-\mu}{\gamma(s) 3^{n}\left[3 \gamma(s)[1-\beta(1-2 \alpha)]-\beta\left(\lambda+2 \alpha \mu+\frac{1}{\beta}\right)\right]} \\
-\sigma\left\{\frac{\gamma(s)(1-\beta)+\beta[2 \alpha(\gamma(s)-\mu)-\lambda]-\mu}{\gamma(s) 2^{n}\left[2 \gamma(s)[1-\beta(1-2 \alpha)]-\beta\left(\lambda+2 \alpha \mu+\frac{1}{\beta}\right)\right]}\right\}^{2} .
\end{gathered}
$$


Set

$$
\begin{gathered}
B_{1}=\left\{\gamma(s) 3^{n}\left[3 \gamma(s)[1-\beta(1-2 \alpha)]-\beta\left(\lambda+2 \alpha \mu+\frac{1}{\beta}\right)\right]\right\} ; \text { and } \\
B_{2}=\left\{\gamma(s) 2^{n}\left[2 \gamma(s)[1-\beta(1-2 \alpha)]-\beta\left(\lambda+2 \alpha \mu+\frac{1}{\beta}\right)\right]\right\} \\
B_{3}=\{(\gamma(s)(1-\beta)+\beta[2 \alpha(\gamma(s)-\mu)-\lambda]-\mu)\} .
\end{gathered}
$$

Thus,

$$
\left|a_{3}-\sigma a_{2}^{2}\right| \leq \frac{B_{3} B_{2}^{2}-\sigma B_{3}^{2} B_{1}}{B_{1} B_{2}^{2}}=\frac{B_{3}\left[B_{2}^{2}-\sigma B_{3} B_{1}\right]}{B_{1} B_{2}^{2}}
$$

which completes the proof.

Radius Properties for class $T_{\gamma}(\lambda, \beta, \alpha, \mu)$.

We now obtain the radii of starlikeness, convexity and close to convexity in this section as follows:

Theorem 3.3 (Starlikeness): Let the function $f_{\gamma}(z)$ defined by (1.1) be in the class $T_{\gamma}(\lambda, \beta, \alpha, \mu)$; then $f_{\gamma}(z)$ is starlike of order $\sigma(0 \leq \delta<1)$ in $|z|<r_{1}$, where

$r_{1}=\inf _{k}\left\{\frac{(1-\delta) \gamma(s) k^{n}\left[\gamma(s) k[1-\beta(1-2 \alpha)]-\beta\left(\lambda+2 \alpha \mu+\frac{1}{\beta}\right)\right]}{[\gamma(s)(1-\beta)+\beta[2 \alpha(\gamma(s)-\mu)-\lambda]-\mu] \gamma(s)(k-\delta)}\right\}^{\frac{1}{k-1}} ; k \geq 2$

The result is sharp for

$$
f_{\gamma}(z)=z-\frac{\gamma(s)(1-\beta)+\beta[2 \alpha(\gamma(s)-\mu)-\lambda]-\mu}{\gamma(s) k^{n}\left[\gamma(s) k[1-\beta(1-2 \alpha)]-\beta\left(\lambda+2 \alpha \mu+\frac{1}{\beta}\right)\right]} z^{k}, \quad k \geq 2 .
$$

Proof: It suffices to show that $\left|\frac{z f^{\prime}(z)}{f(z)}-1\right|<1-\delta$.

That is,

$$
\begin{gathered}
\left|\frac{z f_{\gamma}^{\prime}(z)}{f_{\gamma}}-1\right|=\left|\frac{z-\sum_{k=2}^{\infty} k \gamma(s) a_{k} z^{k}-z+\sum_{k=2}^{\infty} \gamma(s) a_{k} z^{k}}{z-\sum_{k=2}^{\infty} \gamma(s) a_{k} z^{k}}\right| \\
\left|\frac{-\sum_{k=2}^{\infty} \gamma(s)(k-1) a_{k} z^{k-1}}{1-\sum_{k=2}^{\infty} \gamma(s) a_{k} z^{k-1}}\right| \leq \frac{\sum_{k=2}^{\infty} \gamma(s)(k-1) a_{k}|z|^{k-1}}{\left(1-\sum_{k=2}^{\infty} \gamma(s) a_{k}|z|^{k-1}\right)}<1-\delta
\end{gathered}
$$




$$
\begin{gathered}
\sum_{k=2}^{\infty} \gamma(s)(k-1) a_{k}|z|^{k-1} \leq(1-\delta)\left(1-\sum_{k=2}^{\infty} \gamma(s) a_{k}|z|^{k-1}\right) \\
\sum_{k=2}^{\infty} \gamma(s)(k-1) a_{k}|z|^{k-1} \leq(1-\delta)-\sum_{k=2}^{\infty} \gamma(s)(1-\delta) a_{k}|z|^{k-1} \\
\sum_{k=2}^{\infty} \gamma(s)(k-1+1-\delta) a_{k}|z|^{k-1} \leq(1-\delta) \\
\sum_{k=2}^{\infty} \gamma(s)(k+\delta) a_{k}|z|^{k-1} \leq(1-\delta) \\
\sum_{k=2}^{\infty} \gamma(s)\left(\frac{k-\delta}{1-\delta}\right) a_{k}|z|^{k-1} \leq 1
\end{gathered}
$$

Hence by Lemma 2.1, the above inequality holds if

$$
\sum_{k=2}^{\infty} \gamma(s) \frac{k-\delta}{1-\delta} a_{k}|z|^{k-1} \leq 1
$$

It follows that

$$
\begin{gathered}
\sum_{k=2}^{\infty} \gamma(s) \frac{(k-\delta)|z|^{k-1}}{(1-\delta)} \leq \frac{1}{a_{k}} \\
\frac{\gamma(s)(k-\delta)|z|^{k-1}}{(1-\delta)} \leq \frac{\gamma(s) k^{n}\left[\gamma(s) k[1-\beta(1-2 \alpha)]-\beta\left(\lambda+2 \alpha \mu+\frac{1}{\beta}\right)\right]}{\gamma(s)(1-\beta)+\beta[2 \alpha(\gamma(s)-\mu)-\lambda]-\mu} ; \quad(k=2,3, \ldots)
\end{gathered}
$$

By multiplying both sides by the inverse of $\frac{\gamma(s)(k-\delta)}{(1-\delta)}$, we have that

$$
|z|^{k-1} \leq \frac{(1-\delta) \gamma(s) k^{n}\left[\gamma(s) k[1-\beta(1-2 \alpha)]-\beta\left(\lambda+2 \alpha \mu+\frac{1}{\beta}\right)\right]}{[\gamma(s)(1-\beta)+\beta[2 \alpha(\gamma(s)-\mu)-\lambda]-\mu] \gamma(s)(k-\delta)} .
$$

We find the $(k-1)$ th root of both sides, so that $|z| \leq\left\{\frac{(1-\delta) \gamma(s) k^{n}\left[\gamma(s) k[1-\beta(1-2 \alpha)]-\beta\left(\lambda+2 \alpha \mu+\frac{1}{\beta}\right)\right]}{\left\{\gamma^{2}(s)(1-\beta)+\gamma(s) \beta[2 \alpha(\gamma(s)-\mu)-\lambda]-\gamma(s) \mu\right\} \gamma(s)(k-\delta)}\right\}^{\frac{1}{k-1}}$ where $|z|<r_{1}$.

Thus,

$$
r_{1}=\inf _{k}\left\{\frac{(1-\delta) \gamma(s) k^{n}\left[\gamma(s) k[1-\beta(1-2 \alpha)]-\beta\left(\lambda+2 \alpha \mu+\frac{1}{\beta}\right)\right]}{[\gamma(s)(1-\beta)+\beta[2 \alpha(\gamma(s)-\mu)-\lambda]-\mu] \gamma(s)(k-\delta)}\right\}^{\frac{1}{k-1}} ; k \geq 2
$$

which completes the proof. 
Theorem 3.4 (Convexity): Let the function $f_{\gamma}(z)$ defined by (1.1) be in the class $T_{\gamma}(\lambda, \beta, \alpha, \mu)$; then $f_{\gamma}(z)$ is convex of order $\delta(0 \leq \delta<1)$ in $|z|<r_{2}$, where

$r_{2}=\inf _{k}\left\{\frac{(1-\delta) \gamma(s) k^{n}\left[\gamma(s) k[1-\beta(1-2 \alpha)]-\beta\left(\lambda+2 \alpha \mu+\frac{1}{\beta}\right)\right]}{k(k-\delta) \gamma(s)[\gamma(s)(1-\beta)+\beta[2 \alpha(\gamma(s)-\mu)-\lambda]-\mu]}\right\}^{\frac{1}{k-1}} ; k \geq 2$

The result is sharp for

$$
f_{\gamma}(z)=z-\frac{\gamma(s)(1-\beta)+\beta[2 \alpha(\gamma(s)-\mu)-\lambda]-\mu}{\gamma(s) k^{n}\left[\gamma(s) k[1-\beta(1-2 \alpha)]-\beta\left(\lambda+2 \alpha \mu+\frac{1}{\beta}\right)\right]} z^{k}, \quad k \geq 2 .
$$

Proof: It suffices to show that $\left|\frac{z f_{\gamma}^{\prime \prime}(z)}{f_{\gamma}^{\prime}(z)}\right|<1-\delta,|z|<r_{2}$.

Since $\left|\frac{z f_{\gamma}^{\prime \prime}(z)}{f_{\gamma}^{\prime}(z)}\right|=\left|\frac{\sum_{k=2}^{\infty} \gamma(s) k(k-1) a_{k} z^{k-1}}{1-\sum_{k=2}^{\infty} k \gamma(s) a_{k} z^{k-1}}\right| \leq \frac{\sum_{k=2}^{\infty} \gamma(s) k(k-1) a_{k}|z|^{k-1}}{1-\sum_{k=2}^{\infty} \gamma(s) k a_{k}|z|^{k-1}}<1-\delta$

To prove the Theorem, we must show that

$$
\begin{gathered}
\frac{\sum_{k=2}^{\infty} \gamma(s) k(k-1) a_{k}|z|^{k-1}}{1-\sum_{k=2}^{\infty} \gamma(s) k a_{k}|z|^{k-1}} \leq 1-\delta \\
\sum_{k=2}^{\infty} \gamma(s) k(k-\delta) a_{k}|z|^{k-1} \leq 1-\delta .
\end{gathered}
$$

And by Lemma 2.1, we obtain

$$
|z|^{k-1} \leq \frac{(1-\delta) \gamma(s) k^{n}\left[\gamma(s) k[1-\beta(1-2 \alpha)]-\beta\left(\lambda+2 \alpha \mu+\frac{1}{\beta}\right)\right]}{k(k-\delta) \gamma(s)[\gamma(s)(1-\beta)+\beta[2 \alpha(\gamma(s)-\mu)-\lambda]-\mu]}
$$

or

$$
r_{2}=\inf _{k}\left\{\frac{(1-\delta) \gamma(s) k^{n}\left[\gamma(s) k[1-\beta(1-2 \alpha)]-\beta\left(\lambda+2 \alpha \mu+\frac{1}{\beta}\right)\right]}{k(k-\delta) \gamma(s)[\gamma(s) \gamma(s)(1-\beta)+\beta[2 \alpha(\gamma(s)-\mu)-\lambda]-\mu]}\right\}^{\frac{1}{k-1}}
$$

which completes the proof. 
Theorem 3.5 (Close-to-convex): Let the function $f_{\gamma}(z)$ defined by (1.1) be in the class $T_{\gamma}(\lambda, \beta, \alpha, \mu)$. Then $f_{\gamma}(z)$ is closed-to-convex of order $\delta(0 \leq \delta<1)$ in $|z|<r_{3}$, where

$$
r_{3} \leq\left\{\frac{(1-\delta) \gamma(s) k^{n}\left[\gamma(s) k[1-\beta(1-2 \alpha)]-\beta\left(\lambda+2 \alpha \mu+\frac{1}{\beta}\right)\right]}{k \gamma(s)[\gamma(s)(1-\beta)+\beta[2 \alpha(\gamma(s)-\mu)-\lambda]-\mu]}\right\}^{\frac{1}{k-1}} ; k \geq 2 .
$$

The result is sharp

$$
f_{\gamma}(z)=z-\frac{\gamma(s)(1-\beta)+\beta[2 \alpha(\gamma(s)-\mu)-\lambda]-\mu}{\gamma(s) k^{n}\left[\gamma(s) k[1-\beta(1-2 \alpha)]-\beta\left(\lambda+2 \alpha \mu+\frac{1}{\beta}\right)\right]} z^{k}, \quad k \geq 2 .
$$

Proof: It suffices to show that $\left|f_{\gamma}^{\prime}(z)-1\right|=1-\delta, \quad(0 \leq \delta<1)$ for $|z|<r_{3}$.

Thus,

$$
\left|f_{\gamma}^{\prime}(z)-1\right|=\left|1-\sum_{k=2}^{\infty} k \gamma(s) a_{k} z^{k-1}-1\right|=\left|-\sum_{k=2}^{\infty} k \gamma(s) a_{k} z^{k-1}\right| \leq\left|\sum_{k=2}^{\infty} k \gamma(s) a_{k} z^{k-1}\right|
$$

Since $\left|f_{\gamma}^{\prime}(z)-1\right| \leq \sum_{k=2}^{\infty} \gamma(s) k a_{k}\left|z^{k-1}\right| \leq 1-\delta$ if we divide both by $(1-\delta)$, then,

$$
\begin{gathered}
\sum_{k=2}^{\infty} \gamma(s)\left(\frac{k}{1-\delta}\right) a_{k}\left|z^{k-1}\right| \leq 1 \\
\sum_{k=2}^{\infty} \frac{k \gamma(s)\left|z^{k-1}\right|}{(1-\delta} \leq \frac{1}{a_{k}}
\end{gathered}
$$

By coefficient estimates of $f_{\gamma}(z) \in T_{\gamma}(\lambda, \beta, \alpha, \mu)$ given by Lemma 2.1 above, (3.5) holds if

$$
\begin{aligned}
& \sum_{k=2}^{\infty} \frac{k \gamma(s)|z|^{k-1}}{(1-\delta)} \leq \frac{\gamma(s) k^{n}\left[\gamma(s) k[1-\beta(1-2 \alpha)]-\beta\left(\lambda+2 \alpha \mu+\frac{1}{\beta}\right)\right]}{\gamma(s)(1-\beta)+\beta[2 \alpha(\gamma(s)-\mu)-\lambda]-\mu} ; k \geq 2 . \\
& |z| \leq\left\{\frac{(1-\delta) \gamma(s) k^{n}\left[\gamma(s) k[1-\beta(1-2 \alpha)]-\beta\left(\lambda+2 \alpha \mu+\frac{1}{\beta}\right)\right]}{k \gamma(s)[\gamma(s)(1-\beta)+\beta[2 \alpha(\gamma(s)-\mu)-\lambda]-\mu]}\right\}^{\frac{1}{k-1}} ; k \geq 2 .
\end{aligned}
$$

Hence,

$$
r_{3} \leq\left\{\frac{(1-\delta) \gamma(s) k^{n}\left[\gamma(s) k[1-\beta(1-2 \alpha)]-\beta\left(\lambda+2 \alpha \mu+\frac{1}{\beta}\right)\right]}{k\left\{\gamma^{2}(s)(1-\beta)+\gamma(s) \beta[2 \alpha(\gamma(s)-\mu)-\lambda]-\gamma(s) \mu\right\}}\right\}^{\frac{1}{k-1}} ; k \geq 2 .
$$


Neighbourhood Property for class $T_{\gamma}(\lambda, \beta, \alpha, \mu)$.

We define the $(n, \varsigma)$-neighborhood of a function $f_{\gamma} \in T_{\gamma}$ by

$$
N_{(n, \varsigma)}(e)=\left\{g_{\gamma}: g_{\gamma} \in T_{\gamma}, g_{\gamma}(z)=z-\sum_{k=t+1}^{\infty} \gamma(s) b_{k} z^{k} \text { and } \sum_{k=2}^{\infty} \gamma(s) k\left|b_{k}\right| \leq \varsigma\right\}
$$

And for the identity function $e_{\gamma}(z)$ defined by (1.3), we have the following result.

Let $T_{\gamma}$ denotes the class of functions $f_{\gamma}(z)$ defined by (1.1) which is analytic in the open unit disk

$U=\{|z|: z \in C$ and $|z|<1\}$.

Theorem 3.6 Neighbourhood Property for class $T_{\gamma}(\lambda, \beta, \alpha, \mu)$.

Let

$$
\varsigma=\frac{\gamma(s)(1-\beta)+\beta[2 \alpha(\gamma(s)-\mu)-\lambda]-\mu}{\gamma(s) k^{n}\left[\gamma(s) k[1-\beta(1-2 \alpha)]-\beta\left(\lambda+2 \alpha \mu+\frac{1}{\beta}\right)\right]}
$$

Then $T_{\gamma}(\lambda, \beta, \alpha, \mu) \subset N_{(n, \varsigma)} e(z)$.

Proof: Suppose $f_{\gamma} \in T_{\gamma}(\lambda, \beta, \alpha, \mu)$, then from Lemma 2.1, we have that

$$
\begin{gathered}
\sum_{k=2}^{\infty} \gamma(s) k^{n}\left[\gamma(s) k[1-\beta(1-2 \alpha)]-\beta\left(\lambda+2 \alpha \mu+\frac{1}{\beta}\right)\right]\left|a_{k}\right| \\
<\gamma(s)(1-\beta)+\beta[2 \alpha(\gamma(s)-\mu)-\lambda]-\mu
\end{gathered}
$$

and thus for $k=2$, we have that

$$
\begin{gathered}
\gamma(s) 2^{n}\left[\gamma(s) 2[1-\beta(1-2 \alpha)]-\beta\left(\lambda+2 \alpha \mu+\frac{1}{\beta}\right)\right]\left|a_{2}\right| \\
\leq \sum_{k=2}^{\infty} \gamma(s) k^{n}\left[\gamma(s) k[1-\beta(1-2 \alpha)]-\beta\left(\lambda+2 \alpha \mu+\frac{1}{\beta}\right)\right]\left|a_{k}\right| \\
<\gamma(s)(1-\beta)+\beta[2 \alpha(\gamma(s)-\mu)-\lambda]-\mu \\
\sum_{k=2}^{\infty} \gamma(s)\left|a_{k}\right| \leq \frac{\gamma(s)(1-\beta)+\beta[2 \alpha(\gamma(s)-\mu)-\lambda]-\mu}{\gamma(s) k^{n}\left[\gamma(s) k[1-\beta(1-2 \alpha)]-\beta\left(\lambda+2 \alpha \mu+\frac{1}{\beta}\right)\right]}
\end{gathered}
$$

But $|z|<r$ which implies that

$$
\left|f^{\prime}(z)\right| \leq 1-|z| \sum_{k=2}^{\infty} k \gamma(s) a_{k} \leq 1-r \sum_{k=2}^{\infty} k \gamma(s) a_{k}
$$


It thus follows that

$$
\begin{gathered}
\left|f_{\gamma}^{\prime}(z)\right| \leq 1-r \sum_{k=2}^{\infty} k \gamma(s) a_{k} \leq k \frac{\gamma(s)(1-\beta)+\beta[2 \alpha(\gamma(s)-\mu)-\lambda]-\mu}{\gamma(s) k^{n}\left[\gamma(s) k[1-\beta(1-2 \alpha)]-\beta\left(\lambda+2 \alpha \mu+\frac{1}{\beta}\right)\right] k^{n}} \\
\Rightarrow \sum_{k=2}^{\infty} k \gamma(s) a_{k} \leq \sum_{k=2}^{\infty} \gamma(s) a_{k} \\
\leq \frac{\gamma(s)(1-\beta)+\beta[2 \alpha(\gamma(s)-\mu)-\lambda]-\mu}{\gamma(s) k^{n}\left[\gamma(s) k[1-\beta(1-2 \alpha)]-\beta\left(\lambda+2 \alpha \mu+\frac{1}{\beta}\right)\right] k^{n}}=\varsigma \text { by equation }
\end{gathered}
$$

Thus, $f_{\gamma} \in N_{(n, \varsigma)}(e)$.

Theorem 3.7 Convolution Property for class $T_{\gamma}(\lambda, \beta, \alpha, \mu)$.

Let $f_{\gamma}(z)$ and $g_{\gamma}(z)$ defined by (1.1) and (1.2)) respectively be members of $T_{\gamma}(\lambda, \beta, \alpha, \mu)$ such that $h_{\gamma}(z)=z-\sum_{k=2}^{\infty} \gamma(s) a_{k} b_{k} z^{k}$ where $a_{k} b_{k} \geq 0$ as defined by (1.4).

Then, $h_{\gamma}(z)$ is in the subclass of $T_{\gamma}(\lambda, \beta, \alpha, \mu)$ where

$$
\begin{gathered}
\frac{\gamma(s)(1-\beta)+\beta[2 \alpha(\gamma(s)-\mu)-\lambda]-\mu_{1}}{\gamma(s) k^{n}\left[\gamma(s) k[1-\beta(1-2 \alpha)]-\beta\left(\lambda+2 \alpha \mu+\frac{1}{\beta}\right)\right]} \\
\leq \frac{\gamma(s)(1-\beta)+\beta[2 \alpha(\gamma(s)-\mu)-\lambda]-\mu_{1}}{\gamma(s)(1-\beta)+\beta[2 \alpha(\gamma(s)-\mu)-\lambda]-\mu_{2}}
\end{gathered}
$$

Proof: From Lemma 2.1,

$$
\sum_{k=2}^{\infty} \frac{\gamma(s) k^{n}\left[\gamma(s) k[1-\beta(1-2 \alpha)]-\beta\left(\lambda+2 \alpha \mu+\frac{1}{\beta}\right)\right]}{\gamma(s)(1-\beta)+\beta[2 \alpha(\gamma(s)-\mu)-\lambda]-\mu_{1}} a_{k} \leq 1
$$

Similarly,

$$
\sum_{k=2}^{\infty} \frac{\gamma(s) k^{n}\left[\gamma(s) k[1-\beta(1-2 \alpha)]-\beta\left(\lambda+2 \alpha \mu+\frac{1}{\beta}\right)\right]}{\gamma(s)(1-\beta)+\beta[2 \alpha(\gamma(s)-\mu)-\lambda]-\mu_{1}} b_{k} \leq 1
$$

We however need to determine the largest $\mu_{2}$ such that

$$
\sum_{k=2}^{\infty} \frac{\gamma(s) k^{n}\left[\gamma(s) k[1-\beta(1-2 \alpha)]-\beta\left(\lambda+2 \alpha \mu+\frac{1}{\beta}\right)\right]}{\gamma(s)(1-\beta)+\beta[2 \alpha(\gamma(s)-\mu)-\lambda]-\mu_{2}} a_{k} b_{k} \leq 1
$$


By Cauchy-Schwarz inequality,

$$
\sum_{k=2}^{\infty}\left|a_{k} b_{k}\right| \leq \sqrt{\sum_{k=2}^{\infty}\left|a_{k}\right|^{2}} \sqrt{\sum_{k=2}^{\infty}\left|b_{k}\right|^{2}}
$$

Using the Cauchy-Schwarz inequality, we obtain

$$
\sum_{k=2}^{\infty} \frac{\gamma(s) k^{n}\left[\gamma(s) k[1-\beta(1-2 \alpha)]-\beta\left(\lambda+2 \alpha \mu+\frac{1}{\beta}\right)\right]}{\gamma(s)(1-\beta)+\beta[2 \alpha(\gamma(s)-\mu)-\lambda]-\mu_{1}} \sqrt{a_{k} b_{k}} \leq 1
$$

It suffices to show what

$$
\begin{gathered}
\frac{\gamma(s) k^{n}\left[\gamma(s) k[1-\beta(1-2 \alpha)]-\beta\left(\lambda+2 \alpha \mu+\frac{1}{\beta}\right)\right]}{\gamma(s)(1-\beta)+\beta[2 \alpha(\gamma(s)-\mu)-\lambda]-\mu_{2}} a_{k} b_{k} \\
\leq \sum_{k=2}^{\infty} \frac{\gamma(s) k^{n}\left[\gamma(s) k[1-\beta(1-2 \alpha)]-\beta\left(\lambda+2 \alpha \mu+\frac{1}{\beta}\right)\right]}{\gamma(s)(1-\beta)+\beta[2 \alpha(\gamma(s)-\mu)-\lambda]-\mu_{1}} \sqrt{a_{k} b_{k}} \leq 1
\end{gathered}
$$

equivalently,

$$
\begin{gathered}
\sqrt{a_{k} b_{k}} \leq \\
\frac{\gamma(s) k^{n}\left[\gamma(s) k[1-\beta(1-2 \alpha)]-\beta\left(\lambda+2 \alpha \mu+\frac{1}{\beta}\right)\right]}{\gamma(s)(1-\beta)+\beta[2 \alpha(\gamma(s)-\mu)-\lambda]-\mu_{1}} \times \\
\frac{\gamma(s)(1-\beta)+\beta[2 \alpha(\gamma(s)-\mu)-\lambda]-\mu_{2}}{\gamma(s) k^{n}\left[\gamma(s) k[1-\beta(1-2 \alpha)]-\beta\left(\lambda+2 \alpha \mu+\frac{1}{\beta}\right)\right]} \leq \\
\frac{\gamma(s)(1-\beta)+\beta[2 \alpha(\gamma(s)-\mu)-\lambda]-\mu_{1}}{\gamma(s)(1-\beta)+\beta[2 \alpha(\gamma(s)-\mu)-\lambda]-\mu_{2}}
\end{gathered}
$$

But from (3.12) we have

$$
\sqrt{a_{k} b_{k}} \leq \frac{\gamma(s)(1-\beta)+\beta[2 \alpha(\gamma(s)-\mu)-\lambda]-\mu_{1}}{\gamma(s) k^{n}\left[\gamma(s) k[1-\beta(1-2 \alpha)]-\beta\left(\lambda+2 \alpha \mu+\frac{1}{\beta}\right)\right]} .
$$

Consequently, we show that

$$
\begin{gathered}
\frac{\gamma(s)(1-\beta)+\beta[2 \alpha(\gamma(s)-\mu)-\lambda]-\mu_{1}}{\gamma(s) k^{n}\left[\gamma(s) k[1-\beta(1-2 \alpha)]-\beta\left(\lambda+2 \alpha \mu+\frac{1}{\beta}\right)\right]} \\
\leq \frac{\gamma(s)(1-\beta)+\beta[2 \alpha(\gamma(s)-\mu)-\lambda]-\mu_{1}}{\gamma(s)(1-\beta)+\beta[2 \alpha(\gamma(s)-\mu)-\lambda]-\mu_{2}} .
\end{gathered}
$$

Equivalently,

$\frac{\left[\gamma(s)(1-\beta)+\beta[2 \alpha(\gamma(s)-\mu)-\lambda]-\mu_{1}\right]\left[\gamma(s)(1-\beta)+\beta[2 \alpha(\gamma(s)-\mu)-\lambda]-\mu_{2}\right]}{\gamma(s) k^{n}\left[\gamma(s) k[1-\beta(1-2 \alpha)]-\beta\left(\lambda+2 \alpha \mu+\frac{1}{\beta}\right)\right]\left[\gamma(s)(1-\beta)+\beta[2 \alpha(\gamma(s)-\mu)-\lambda]-\mu_{1}\right]} \leq 1$. 


\section{REFERENCES}

[1] Fadipe-Joseph O. A., Moses B.O. and Oluwayemi M. O. (2016). Certain New Classes of analytic functions defined by using sigmoid function. Advances in Mathematics: Scientific Journal, 5, 83-89.

[2] Khan M. G., Ahmed B., Murugusundaramoorthy G., Chinram R. and Mashwani W. K. (2020). Application of Modified Sigmoid Function to a Class of Starlike Functions, Journal of Function Spaces, 2020, 1-8, https://doi.org/10.1155/2020/8844814.

[3] Murugusundaramoorthy G. and Janani T. (2015). Sigmoid Function in the Space of Univalent $\lambda$-Pseudo Starlike Functions, International Journal of Pure and Applied Mathematics, 101(1), 33 - 41.

[4] Oluwayemi M. O., Fadipe-Joseph Olubunmi A. and Ezeafulukwe U. A. (2019). Certain subclass of univalent functions involving modified sigmoid function, 14th International Symposium on Geometric Function Theory and Applications, IOP Conference Series: Journal of Physics: Conference Series 1212 (2019) 012005 IOP Publishing, doi:10.1088/1742-6596/1212/1/012005. 
